\title{
Certain classes of bi-univalent functions associated with the Horadam polynomials
}

\author{
Halit Orhan \\ Department of Mathematics, \\ Faculty of Science, Ataturk University, \\ 25240 Erzurum, Turkey \\ email: orhanhalit607@gmail.com
}

Paduvalapattana Kempegowda Mamatha Department of Mathematics, R. V. College of Engineering, Bangalore-560 059 Karnataka, India email: mamatharaviv@gmail.com

Nanjundan Magesh

Post-Graduate and Research

Department of Mathematics, Government Arts College for Men, Krishnagiri 635001, Tamilnadu, India email: nmagi_2000@yahoo.co.in
Sondekola Rudra Swamy

Department of Computer Science and Engineering,

R. V. College of Engineering, Bangalore-560 059 Karnataka, India email: mailtoswamy@rediffmail.com

\begin{abstract}
In this paper we consider two subclasses of bi-univalent functions defined by the Horadam polynomials. Further, we obtain coefficient estimates for the defined classes.
\end{abstract}

2010 Mathematics Subject Classification: 11B39, 30C45,33C45, 30C50, 33C05.

Key words and phrases: univalent functions, bi-univalent functions, bi-convex functions, bi-starlike functions, Fekete-Szegő inequality, Horadam polynomials 


\section{Introduction}

Let $\mathcal{A}$ denote the class of functions of the form

$$
f(z)=z+\sum_{n=2}^{\infty} a_{n} z^{n}
$$

which are analytic in the open unit disk $\Delta=\{z: z \in \mathbb{C}$ and $|z|<1\}$. Further, by $\mathcal{S}$ we shall denote the class of all functions in $\mathcal{A}$ which are univalent in $\Delta$.

It is well known that every function $f \in \mathcal{S}$ has an inverse $f^{-1}$, defined by

$$
f^{-1}(f(z))=z \quad(z \in \Delta)
$$

and

$$
f\left(f^{-1}(w)\right)=w \quad\left(|w|<r_{0}(f) ; r_{0}(f) \geqq \frac{1}{4}\right),
$$

where

$$
f^{-1}(w)=w-a_{2} w^{2}+\left(2 a_{2}^{2}-a_{3}\right) w^{3}-\left(5 a_{2}^{3}-5 a_{2} a_{3}+a_{4}\right) w^{4}+\cdots .
$$

A function $\mathrm{f} \in \mathcal{A}$ is said to be bi-univalent in $\Delta$ if both the function $\mathrm{f}$ and its inverse $f^{-1}$ are univalent in $\Delta$. Let $\Sigma$ denote the class of bi-univalent functions in $\Delta$ given by (1).

In 2010, Srivastava et al. [28] revived the study of bi-univalent functions by their pioneering work on the study of coefficient problems. Various subclasses of the bi-univalent function class $\Sigma$ were introduced and non-sharp estimates on the first two coefficients $\left|a_{2}\right|$ and $\left|a_{3}\right|$ in the Taylor-Maclaurin series expansion (1) were found in the very recent investigations (see, for example, [1, 2, 3, 4, $5,6,7,8,9,10,12,13,16,17,18,19,20,21,22,23,24,25,26,27,29,30])$ and including the references therein. The afore-cited all these papers on the subject were actually motivated by the work of Srivastava et al. [28]. However, the problem to find the coefficient bounds on $\left|a_{n}\right|(n=3,4, \cdots)$ for functions $f \in \Sigma$ is still an open problem.

For analytic functions $f$ and $g$ in $\Delta, f$ is said to be subordinate to $g$ if there exists an analytic function $w$ such that

$$
w(0)=0, \quad|w(z)|<1 \quad \text { and } \quad f(z)=g(w(z)) \quad(z \in \Delta) .
$$

This subordination will be denoted here by

$$
f \prec g \quad(z \in \Delta)
$$


or, conventionally, by

$$
\mathrm{f}(z) \prec \mathrm{g}(z) \quad(z \in \Delta) .
$$

In particular, when $\mathrm{g}$ is univalent in $\Delta$,

$$
\mathrm{f} \prec \mathrm{g} \quad(z \in \Delta) \Leftrightarrow \mathrm{f}(0)=\mathrm{g}(0) \text { and } \mathrm{f}(\Delta) \subset \mathrm{g}(\Delta) .
$$

The Horadam polynomials $h_{n}(x, a, b ; p, q)$, or briefly $h_{n}(x)$ are given by the following recurrence relation (see $[14,15])$ :

$h_{1}(x)=a, \quad h_{2}(x)=b x \quad$ and $\quad h_{n}(x)=p x h_{n-1}(x)+q h_{n-2}(x) \quad(n \geq 3)$

for some real constants $a, b, p$ and $q$.

The generating function of the Horadam polynomials $h_{n}(x)$ (see [15]) is given by

$$
\Pi(x, z):=\sum_{n=1}^{\infty} h_{n}(x) z^{n-1}=\frac{a+(b-a p) x z}{1-p x z-q z^{2}} .
$$

Here, and in what follows, the argument $x \in \mathbb{R}$ is independent of the argument $z \in \mathbb{C}$; that is, $x \neq \mathfrak{R}(z)$.

Note that for particular values of $a, b, p$ and $q$, the Horadam polynomial $h_{n}(x)$ leads to various polynomials, among those, we list a few cases here (see, $[14,15]$ for more details):

1. For $a=b=p=q=1$, we have the Fibonacci polynomials $F_{n}(x)$.

2. For $a=2$ and $b=p=q=1$, we obtain the Lucas polynomials $L_{n}(x)$.

3. For $a=q=1$ and $b=p=2$, we get the Pell polynomials $P_{n}(x)$.

4. For $a=b=p=2$ and $q=1$, we attain the Pell-Lucas polynomials $Q_{n}(x)$.

5. For $\mathrm{a}=\mathrm{b}=1, \mathrm{p}=2$ and $\mathrm{q}=-1$, we have the Chebyshev polynomials $\mathrm{T}_{\mathrm{n}}(x)$ of the first kind

6. For $a=1, b=p=2$ and $q=-1$, we obtain the Chebyshev polynomials $\mathrm{U}_{\mathrm{n}}(\mathrm{x})$ of the second kind.

Abirami et al. [1] considered bi- Mocanu - convex functions and bi- $\mu-$ starlike functions to discuss initial coefficient estimations of Taylor-Macularin series which is associated with Horadam polynomials, Abirami et al. [2] discussed coefficient estimates for the classes of $\lambda$-bi-pseudo-starlike and bi-Bazilevič 
functions using Horadam polynomial, Alamoush [3, 4] defined subclasses of bi-starlike and bi-convex functions involving the Poisson distribution series involving Horadam polynomials and a class of bi-univalent functions associated with Horadam polynomials respectively and obtained initial coefficient estimates, Altınkaya and Yalçın [7, 8] obtained coefficient estimates for Pascutype bi-univalent functions and for the class of linear combinations of biunivalent functions by means of $(p, q)$-Lucas polynomials respectively, Aouf et al. [10] discussed initial coefficient estimates for general class of pascu-type bi-univalent functions of complex order defined by q-Sălăgean operator and associated with Chebyshev polynomials, Awolere and Oladipo [11] found initial coefficients of bi-univalent functions defined by sigmoid functions involving pseudo-starlikeness associated with Chebyshev polynomials, Naeem et al. [18] considered a general class of bi-Bazilevič type functions associated with Faber polynomial to discuss n-th coefficients estimates, Magesh and Bulut [19] discussed Chebyshev polynomial coefficient estimates for a class of analytic bi-univalent functions related to pseudo-starlike functions, Orhan et al. [21] discussed initial estimates and Fekete-Szegö bounds for bi-Bazilevič functions related to shell-like curves, Sakar and Aydogan [23] obtained initial bounds for the class of generalized Sălăgean type bi- $\alpha$ - convex functions of complex order associated with the Horadam polynomials, Singh et al. [24] found coefficient estimates for bi- $\alpha$-convex functions defined by generalized Sãlãgean operator related to shell-like curves connected with Fibonacci numbers, Srivastava et al. [25] introduced a technique by defining a new class bi-univalent functions associated with the Horadam polynomials to discuss the coefficient estimates, Srivastava et al. [27] gave a direction to study the Faber polynomial coefficient estimates for bi-univalent functions defined by the Tremblay fractional derivative operator, Srivastava et al. [29] obtained general coefficient $\left|a_{n}\right|$ for a general class analytic and bi-univalent functions defined by using differential subordination and a certain fractional derivative operator associated with Faber polynomial, Wanas and Alina [30] discussed applications of Horadam polynomials on Bazilevič bi-univalent functions by means of subordination and found initial bounds. Motivated in these lines, estimates on initial coefficients of the Taylor-Maclaurin series expansion (1) and Fekete-Szegö inequalities for certain classes of bi-univalent functions defined by means of Horadam polynomials are obtained. The classes introduced in this paper are motivated by the corresponding classes investigated in $[16,20]$. 


\section{Coefficient estimates and Fekete-Szegő inequali- ties}

A function $f \in \mathcal{A}$ of the form (1) belongs to the class $\mathcal{G}_{\Sigma}^{*}(\alpha, x)$ for $0 \leq \alpha \leq 1$ and $z, w \in \Delta$, if the following conditions are satisfied:

$$
\alpha\left(1+\frac{z f^{\prime \prime}(z)}{f^{\prime}(z)}\right)+(1-\alpha) f^{\prime}(z) \prec \Pi(x, z)+1-a
$$

and for $g(w)=f^{-1}(w)$

$$
\alpha\left(1+\frac{w g^{\prime \prime}(w)}{g^{\prime}(w)}\right)+(1-\alpha) g^{\prime}(w) \prec \Pi(x, w)+1-a,
$$

where the real constant $\boldsymbol{a}$ is as in (2).

Remark 1 The classes $\mathcal{K}_{\Sigma}(\mathrm{x})$ and $\mathcal{H}_{\Sigma}(\mathrm{x})$ are defined by $\mathcal{G}_{\Sigma}^{*}(1, x):=\mathcal{K}_{\Sigma}(\mathrm{x})$ and introduced by [1] and $\mathcal{G}_{\Sigma}^{*}(0, x):=\mathcal{H}_{\Sigma}(x)$ introduced by [4] respectively.

For functions in the class $\mathcal{G}_{\Sigma}^{*}(\alpha, x)$, the following coefficient estimates and Fekete-Szegő inequality are obtained.

Theorem 1 Let $f(z)=z+\sum_{n=2}^{\infty} a_{n} z^{n}$ be in the class $\mathcal{G}_{\Sigma}^{*}(\alpha, x)$. Then

$$
\left|a_{2}\right| \leq \frac{|b x| \sqrt{|b x|}}{\sqrt{\left|(3-\alpha) b^{2} x^{2}-4\left(p x^{2} b+q a\right)\right|}}, \quad \text { and } \quad\left|a_{3}\right| \leq \frac{|b x|}{3(\alpha+1)}+\frac{b^{2} x^{2}}{4}
$$

and for $v \in \mathbb{R}$

$$
\left|a_{3}-v a_{2}^{2}\right| \leq \begin{cases}\frac{|b x|}{3 \alpha+3} & \text { if }|v-1| \leq \frac{\left|(3-\alpha) b^{2} x^{2}-4\left(p x^{2} b+q a\right)\right|}{b^{2} x^{2}(3 \alpha+3)} \\ \frac{|b x|^{3}|v-1|}{\left|(3-\alpha) b^{2} x^{2}-4\left(p x^{2} b+q a\right)\right|} & \text { if }|v-1| \geq \frac{\left|(3-\alpha) b^{2} x^{2}-4\left(p x^{2} b+q a\right)\right|}{b^{2} x^{2}(3 \alpha+3)} .\end{cases}
$$

Proof. Let $f \in \mathcal{G}_{\Sigma}^{*}(\alpha, x)$ be given by the Taylor-Maclaurin expansion (1). Then, there are analytic functions $r$ and $s$ such that

$$
\mathrm{r}(0)=0 ; \quad s(0)=0, \quad|r(z)|<1 \quad \text { and } \quad|s(w)|<1 \quad(\forall z, w \in \Delta),
$$

and we can write

$$
\alpha\left(1+\frac{z f^{\prime \prime}(z)}{f^{\prime}(z)}\right)+(1-\alpha) f^{\prime}(z)=\Pi(x, r(z))+1-a
$$


and

$$
\alpha\left(1+\frac{w g^{\prime \prime}(w)}{g^{\prime}(w)}\right)+(1-\alpha) g^{\prime}(w)=\Pi(x, s(w))+1-a .
$$

Equivalently,

$$
\begin{aligned}
\alpha(1 & \left.+\frac{z f^{\prime \prime}(z)}{f^{\prime}(z)}\right)+(1-\alpha) f^{\prime}(z) \\
& =1+h_{1}(x)-a+h_{2}(x) r(z)+h_{3}(x)[r(z)]^{2}+\cdots
\end{aligned}
$$

and

$$
\begin{aligned}
\alpha(1 & \left.+\frac{w g^{\prime \prime}(w)}{g^{\prime}(w)}\right)+(1-\alpha) g^{\prime}(w) \\
& =1+h_{1}(x)-a+h_{2}(x) s(w)+h_{3}(x)[s(w)]^{2}+\cdots
\end{aligned}
$$

From (6) and (7) and in view of (3), we obtain

$$
\begin{aligned}
\alpha(1 & \left.+\frac{z f^{\prime \prime}(z)}{f^{\prime}(z)}\right)+(1-\alpha) f^{\prime}(z) \\
& =1+h_{2}(x) r_{1} z+\left[h_{2}(x) r_{2}+h_{3}(x) r_{1}^{2}\right] z^{2}+\cdots
\end{aligned}
$$

and

$$
\begin{aligned}
\alpha(1 & \left.+\frac{w g^{\prime \prime}(w)}{g^{\prime}(w)}\right)+(1-\alpha) g^{\prime}(w) \\
& =1+h_{2}(x) s_{1} w+\left[h_{2}(x) s_{2}+h_{3}(x) s_{1}^{2}\right] w^{2}+\cdots .
\end{aligned}
$$

If

$$
r(z)=\sum_{n=1}^{\infty} r_{n} z^{n} \quad \text { and } \quad s(w)=\sum_{n=1}^{\infty} s_{n} w^{n},
$$

then it is well known that

$$
\left|r_{n}\right| \leq 1 \quad \text { and } \quad\left|s_{n}\right| \leq 1 \quad(n \in \mathbb{N}) .
$$

Thus upon comparing the corresponding coefficients in (8) and (9), we have

$$
\begin{gathered}
2 a_{2}=h_{2}(x) r_{1} \\
3(\alpha+1) a_{3}-4 a_{2}^{2} \alpha=h_{2}(x) r_{2}+h_{3}(x) r_{1}^{2} \\
-2 a_{2}=h_{2}(x) s_{1}
\end{gathered}
$$


and

$$
2(\alpha+3) a_{2}^{2}-3(\alpha+1) a_{3}=h_{2}(x) s_{2}+h_{3}(x) s_{1}^{2} .
$$

From (10) and (12), we can easily see that

$$
r_{1}=-s_{1}, \quad \text { provided } \quad h_{2}(x)=b x \neq 0
$$

and

$$
\begin{aligned}
8 a_{2}^{2} & =\left(h_{2}(x)\right)^{2}\left(r_{1}{ }^{2}+s_{1}{ }^{2}\right) \\
a_{2}^{2} & =\frac{1}{8}\left(h_{2}(x)\right)^{2}\left(r_{1}{ }^{2}+s_{1}{ }^{2}\right) .
\end{aligned}
$$

If we add (11) to (13), we get

$$
2 a_{2}^{2}(3-\alpha)=\left(r_{2}+s_{2}\right) h_{2}(x)+h_{3}(x)\left(r_{1}{ }^{2}+s_{1}{ }^{2}\right) .
$$

By substituting (15) in (16), we obtain

$$
a_{2}^{2}=\frac{\left(r_{2}+s_{2}\right)\left(h_{2}(x)\right)^{3}}{2(3-\alpha)\left(h_{2}(x)\right)^{2}-8 h_{3}(x)}
$$

and by taking $h_{2}(x)=b x$ and $h_{3}(x)=b p x^{2}+q a$ in (17), it further yields

$$
\left|a_{2}\right| \leq \frac{|b x| \sqrt{|b x|}}{\sqrt{\left|(3-\alpha) b^{2} x^{2}-4\left(p x^{2} b+q a\right)\right|}} .
$$

By subtracting (13) from (11) we get

$$
6(\alpha+1)\left(a_{3}-a_{2}^{2}\right)=\left(r_{2}-s_{2}\right) h_{2}(x)+\left(r_{1}^{2}-s_{1}^{2}\right) h_{3}(x) .
$$

In view of (14), we obtain

$$
a_{3}=\frac{\left(r_{2}-s_{2}\right) h_{2}(x)}{6(\alpha+1)}+a_{2}^{2} .
$$

Then in view of (15), (19) becomes

$$
a_{3}=\frac{\left(r_{2}-s_{2}\right) h_{2}(x)}{6(\alpha+1)}+\frac{1}{8}\left(h_{2}(x)\right)^{2}\left(r_{1}^{2}+s_{1}^{2}\right) \text {. }
$$

Applying (2), we deduce that

$$
\left|a_{3}\right| \leq \frac{|b x|}{3(\alpha+1)}+\frac{b^{2} x^{2}}{4} .
$$


From (19), for $v \in \mathbb{R}$, we write

$$
a_{3}-v a_{2}^{2}=\frac{h_{2}(x)\left(r_{2}-s_{2}\right)}{6(\alpha+1)}+(1-v) a_{2}^{2} .
$$

By substituting (17) in (20), we have

$$
\begin{aligned}
a_{3}-v a_{2}^{2} & =\frac{h_{2}(x)\left(r_{2}-s_{2}\right)}{6(\alpha+1)}+\left(\frac{(1-v)\left(r_{2}+s_{2}\right)\left(h_{2}(x)\right)^{3}}{2(3-\alpha)\left(h_{2}(x)\right)^{2}-8 h_{3}(x)}\right) \\
& =h_{2}(x)\left\{\left(\Lambda_{1}(v, x)+\frac{1}{6(\alpha+1)}\right) r_{2}+\left(\Lambda_{1}(v, x)-\frac{1}{6(\alpha+1)}\right) s_{2}\right\},
\end{aligned}
$$

where

$$
\Lambda_{1}(v, x)=\frac{(1-v)\left[h_{2}(x)\right]^{2}}{2(3-\alpha)\left(h_{2}(x)\right)^{2}-8 h_{3}(x)} .
$$

Hence, in view of (2) we conclude that

$$
\left|a_{3}-v a_{2}^{2}\right| \leq \begin{cases}\frac{\left|h_{2}(x)\right|}{3(\alpha+1)} & ; 0 \leq\left|\Lambda_{1}(\nu, x)\right| \leq \frac{1}{6(\alpha+1)} \\ 2\left|h_{2}(x)\right|\left|\Lambda_{1}(\nu, x)\right| & ;\left|\Lambda_{1}(\nu, x)\right| \geq \frac{1}{6(\alpha+1)}\end{cases}
$$

and in view of (2), it evidently completes the proof of Theorem 1 . Taking $\alpha=1$ in Theorem 1, we have following corollary.

Corollary 1 Let $\mathrm{f}(z)=z+\sum_{n=2}^{\infty} a_{n} z^{\mathfrak{n}}$ be in the class $\mathcal{K}_{\Sigma}(x)$. Then

$$
\left|a_{2}\right| \leq \frac{|b x| \sqrt{|b x|}}{\sqrt{\left|2 b^{2} x^{2}-4\left(p x^{2} b+q a\right)\right|}}, \quad \text { and } \quad\left|a_{3}\right| \leq \frac{|b x|}{6}+\frac{b^{2} x^{2}}{4}
$$

and for $v \in \mathbb{R}$

$$
\left|a_{3}-v a_{2}^{2}\right| \leq \begin{cases}\frac{|b x|}{6} & \text { if }|v-1| \leq \frac{\left|b^{2} x^{2}-2\left(p x^{2} b+q a\right)\right|}{3 b^{2} x^{2}} \\ \frac{|b x|^{3}|v-1|}{\left|2 b^{2} x^{2}-4\left(p x^{2} b+q a\right)\right|} & \text { if }|v-1| \geq \frac{\left|b^{2} x^{2}-2\left(p x^{2} b+q a\right)\right|}{3 b^{2} x^{2}} .\end{cases}
$$


Taking $\alpha=0$ in Theorem 1 , we have following corollary.

Corollary 2 Let $\mathrm{f}(z)=z+\sum_{n=2}^{\infty} a_{n} z^{n}$ be in the class $\mathcal{H}_{\Sigma}(x)$. Then

$$
\left|a_{2}\right| \leq \frac{|b x| \sqrt{|b x|}}{\sqrt{\left|3 b^{2} x^{2}-4\left(p x^{2} b+q a\right)\right|}}, \quad \text { and } \quad\left|a_{3}\right| \leq \frac{|b x|}{3}+\frac{b^{2} x^{2}}{4}
$$

and for $v \in \mathbb{R}$

$$
\left|a_{3}-v a_{2}^{2}\right| \leq \begin{cases}\frac{|b x|}{3} & \text { if }|v-1| \leq \frac{\left|3 b^{2} x^{2}-4\left(p x^{2} b+q a\right)\right|}{3 b^{2} x^{2}} \\ \frac{|b x|^{3}|v-1|}{\left|3 b^{2} x^{2}-4\left(p x^{2} b+q a\right)\right|} & \text { if }|v-1| \geq \frac{\left|3 b^{2} x^{2}-4\left(p x^{2} b+q a\right)\right|}{3 b^{2} x^{2}} .\end{cases}
$$

Next, a function $f \in \mathcal{A}$ of the form (1) belongs to the class $\mathcal{L}_{\Sigma}(x)$ and $z, w \in \Delta$, if the following conditions are satisfied:

$$
\frac{1+\frac{z f^{\prime \prime}(z)}{f^{\prime}(z)}}{\frac{z f^{\prime}(z)}{f(z)}} \prec \Pi(x, z)+1-a
$$

and for $g(w)=f^{-1}(w)$

$$
\frac{1+\frac{w g^{\prime \prime}(w)}{g^{\prime}(w)}}{\frac{w g^{\prime}(w)}{g(w)}} \prec \Pi(x, w)+1-a,
$$

where the real constant $\boldsymbol{a}$ is as in (2).

For functions in the class $\mathcal{L}_{\Sigma}(x)$, the following coefficient estimates and Fekete-Szegő inequality are obtained.

Theorem 2 Let $f(z)=z+\sum_{n=2}^{\infty} a_{n} z^{n}$ be in the class $\mathcal{L}_{\Sigma}(x)$. Then

$$
\left|\mathrm{a}_{2}\right| \leq \frac{|\mathrm{bx}| \sqrt{|\mathrm{bx}|}}{\sqrt{\left|\mathrm{px^{2 }} \mathrm{b}+\mathrm{qa}\right|}}, \quad \text { and } \quad\left|\mathrm{a}_{3}\right| \leq \frac{|\mathrm{bx}|}{4}+\mathrm{b}^{2} \mathrm{x}^{2}
$$


and for $v \in \mathbb{R}$

$$
\left|a_{3}-v a_{2}^{2}\right| \leq\left\{\begin{array}{lll}
\frac{|b x|}{4} & \text { if } & |v-1| \leq \frac{\left|b p x^{2}+a q\right|}{4 b^{2} x^{2}} \\
\frac{|b x|^{3}|v-1|}{\left|b p x^{2}+a q\right|} & \text { if } & |v-1| \geq \frac{\left|b p x^{2}+a q\right|}{4 b^{2} x^{2}} .
\end{array}\right.
$$

Proof. Let $f \in \mathcal{L}_{\Sigma}(x)$ be given by the Taylor-Maclaurin expansion (1). Then, there are analytic functions $r$ and $s$ such that

$$
\mathrm{r}(0)=0 ; \quad \mathrm{s}(0)=0, \quad|\mathrm{r}(z)|<1 \quad \text { and } \quad|\mathrm{s}(w)|<1 \quad(\forall z, w \in \Delta),
$$

and we can write

$$
\frac{1+\frac{z f^{\prime \prime}(z)}{f^{\prime}(z)}}{\frac{z f^{\prime}(z)}{f(z)}}=\Pi(x, r(z))+1-a
$$

and

$$
\frac{1+\frac{w g^{\prime \prime}(w)}{g^{\prime}(w)}}{\frac{w g^{\prime}(w)}{g(w)}}=\Pi(x, s(w))+1-a
$$

Equivalently,

$$
\frac{1+\frac{z f^{\prime \prime}(z)}{f^{\prime}(z)}}{\frac{z f^{\prime}(z)}{f(z)}}=1+h_{1}(x)-a+h_{2}(x) r(z)+h_{3}(x)[r(z)]^{2}+\cdots
$$

and

$$
\frac{1+\frac{w g^{\prime \prime}(w)}{g^{\prime}(w)}}{\frac{w g^{\prime}(w)}{g(w)}}=1+h_{1}(x)-a+h_{2}(x) s(w)+h_{3}(x)[s(w)]^{2}+\cdots
$$

From (24) and (25) and in view of (3), we obtain

$$
\frac{1+\frac{z f^{\prime \prime}(z)}{f^{\prime}(z)}}{\frac{z f^{\prime}(z)}{f(z)}}=1+h_{2}(x) r_{1} z+\left[h_{2}(x) r_{2}+h_{3}(x) r_{1}^{2}\right] z^{2}+\cdots
$$


and

$$
\frac{1+\frac{w g^{\prime \prime}(w)}{g^{\prime}(w)}}{\frac{w g^{\prime}(w)}{g(w)}}=1+h_{2}(x) s_{1} w+\left[h_{2}(x) s_{2}+h_{3}(x) s_{1}^{2}\right] w^{2}+\cdots
$$

If

$$
r(z)=\sum_{n=1}^{\infty} r_{n} z^{n} \quad \text { and } \quad s(w)=\sum_{n=1}^{\infty} s_{n} w^{n},
$$

then it is well known that

$$
\left|r_{n}\right| \leq 1 \quad \text { and } \quad\left|s_{n}\right| \leq 1 \quad(n \in \mathbb{N}) .
$$

Thus upon comparing the corresponding coefficients in (26) and (27), we have

$$
\begin{gathered}
a_{2}=h_{2}(x) r_{1} \\
4\left(a_{3}-a_{2}^{2}\right)=h_{2}(x) r_{2}+h_{3}(x) r_{1}^{2} \\
-a_{2}=h_{2}(x) s_{1}
\end{gathered}
$$

and

$$
4\left(a_{2}^{2}-a_{3}\right)=h_{2}(x) s_{2}+h_{3}(x) s_{1}^{2} .
$$

From (28) and (30), we can easily see that

$$
r_{1}=-s_{1}, \quad \text { provided } \quad h_{2}(x)=b x \neq 0
$$

and

$$
\begin{aligned}
2 a_{2}^{2} & =\left(h_{2}(x)\right)^{2}\left(r_{1}{ }^{2}+s_{1}{ }^{2}\right) \\
a_{2}^{2} & =\frac{1}{2}\left(h_{2}(x)\right)^{2}\left(r_{1}{ }^{2}+s_{1}{ }^{2}\right) .
\end{aligned}
$$

If we add (29) to (31), we get

$$
0=\left(r_{2}+s_{2}\right) h_{2}(x)+h_{3}(x)\left(r_{1}^{2}+s_{1}^{2}\right) .
$$

By substituting (33) in (34), we obtain

$$
a_{2}^{2}=-\frac{\left(r_{2}+s_{2}\right)\left(h_{2}(x)\right)^{3}}{2 h_{3}(x)}
$$


and by taking $h_{2}(x)=b x$ and $h_{3}(x)=b p x^{2}+q a$ in (35), it further yields

$$
\left|a_{2}\right| \leq \frac{|b x| \sqrt{|b x|}}{\sqrt{\left|p x^{2} b+q a\right|}} .
$$

By subtracting (31) from (29) we get

$$
-8\left(a_{2}^{2}-a_{3}\right)=\left(r_{2}-s_{2}\right) h_{2}(x)+\left(r_{1}^{2}-s_{1}^{2}\right) h_{3}(x)
$$

In view of (32), we obtain

$$
a_{3}=\frac{1}{8}\left(r_{2}-s_{2}\right) h_{2}(x)+a_{2}{ }^{2} .
$$

Then in view of (33), (37) becomes

$$
a_{3}=\frac{1}{8}\left(r_{2}-s_{2}\right) h_{2}(x)+\frac{1}{2}\left(h_{2}(x)\right)^{2}\left(r_{1}^{2}+s_{1}^{2}\right) .
$$

Applying (2), we deduce that

$$
\left|a_{3}\right| \leq \frac{|b x|}{4}+b^{2} x^{2}
$$

From (37), for $v \in \mathbb{R}$, we write

$$
a_{3}-v a_{2}^{2}=\frac{1}{8} h_{2}(x)\left(r_{2}-s_{2}\right)+(1-v) a_{2}^{2} .
$$

By substituting (35) in (38), we have

$$
\begin{aligned}
a_{3}-v a_{2}^{2} & =\frac{1}{8} h_{2}(x)\left(r_{2}-s_{2}\right)+\left(\frac{(v-1)\left(r_{2}+s_{2}\right)\left(h_{2}(x)\right)^{3}}{2 h_{3}(x)}\right) \\
& =h_{2}(x)\left\{\left(\Lambda_{2}(v, x)+\frac{1}{8}\right) r_{2}+\left(\Lambda_{2}(v, x)-\frac{1}{8}\right) s_{2}\right\},
\end{aligned}
$$

where

$$
\Lambda_{2}(v, x)=\frac{(v-1)\left(h_{2}(x)\right)^{2}}{2 h_{3}(x)}
$$

Hence, in view of (2) we conclude that

$$
\left|a_{3}-v a_{2}^{2}\right| \leq \begin{cases}\frac{\left|h_{2}(x)\right|}{4} ; & 0 \leq\left|\Lambda_{2}(v, x)\right| \leq \frac{1}{8} \\ 2\left|h_{2}(x)\right|\left|\Lambda_{2}(v, x)\right| ; & \left|\Lambda_{2}(\nu, x)\right| \geq \frac{1}{8}\end{cases}
$$

and in view of (2), it evidently completes the proof of Theorem 2 . 


\section{Acknowledgements}

The authors would like to thank the referee(s) for their constructive suggestions which improved basically the final version of this work.

\section{References}

[1] C. Abirami, N. Magesh and J. Yamini, Initial bounds for certain classes of bi-univalent functions defined by Horadam polynomials, Abstr. Appl. Anal. 2020, Art. ID 7391058, 8 pp.

[2] C. Abirami, N. Magesh, J. Yamini, and N. B. Gatti, Horadam polynomial coefficient estimates for the classes of $\lambda$-bi-pseudo-starlike and biBazilevič functions, J. Anal., (2020), 1-10.

[3] A. G. Alamoush, Certain subclasses of bi-univalent functions involving the Poisson distribution associated with Horadam polynomials, Malaya J. Mat., 7 (2019), no. 4, 618-624.

[4] A. G. Alamoush, On a subclass of bi-univalent functions associated to Horadam polynomials, Int. J. Open Problems Complex Anal., 12 (2020), no. $1,58-65$.

[5] I. Aldawish, T. Al-Hawary and B. A. Frasin, Subclasses of bi-Univalent functions defined by Frasin differential operator, Mathematics, 8 (2020), $783,1-11$.

[6] R. M. Ali, S. K. Lee, V. Ravichandran, S. Supramanian, Coefficient estimates for bi-univalent Ma-Minda starlike and convex functions, Appl. Math. Lett., 25 (2012), no. 3, 344-351.

[7] Ş. Altınkaya and S. Yalçın, On the $(p, q)$-Lucas polynomial coefficient bounds of the bi-univalent function class $\sigma$, Bol. Soc. Mat. Mex., (3) 25 (2019), no. 3, 567-575.

[8] Ş. Altınkaya and S. Yalçın, (p, q)-Lucas polynomials and their applications to bi-univalent functions, Proyecciones, 38 (2019), no. 5, 1093-1105.

[9] O. Al-Refai and M. Ali, General coefficient estimates for bi-univalent functions: a new approach, Turk. J. Math., 44 (2020), 240- 251. 
[10] M. K. Aouf, A. O. Mostafa, R. E. El. Morsy, Coefficient bounds for general class of bi-univalent functions of complex order associated with q-Sălăgean operator and Chebyshev polynomials, Electr. J. Math. Anal. Appl., 8 (2020), no. 2, 251-260.

[11] I. T. Awolere and A. T. Oladipo, Coefficients of bi-univalent functions involving pseudo-starlikeness associated with Chebyshev polynomials, Khayyam J. Math., 5 (2019), no. 1, 140-149.

[12] M. Çă̆lar, E. Deniz, H. M. Srivastava, Second Hankel determinant for certain subclasses of bi-univalent functions, Turk J. Math., 41, (2017), 694-706.

[13] S. M. El-Deeb, T. Bulboaca and B. M. El-Matary, Maclaurin coefficient estimates of bi-Univalent functions connected with the q-derivative, Mathematics, 8 (2020), 418, 1-14.

[14] A. F. Horadam, J. M. Mahon, Pell and Pell-Lucas polynomials, Fibonacci Quart., 23, (1985), 7-20.

[15] T. Hörçum, E. Gökçen Koçer, On some properties of Horadam polynomials, Int Math Forum, 4 (2009), 1243-1252.

[16] A. Y. Lashin, Coefficient estimates for two subclasses of analytic and bi-univalent functions, Ukrainian Math. J., 70 (2019), no. 9, 1484-1492

[17] P. Long, H. Tang, W. Wang, Fekete-Szegö functional problems for certain subclasses of bi-univalent functions involving the Hohlov operator, J. Math. Res. Appl., 40 (2020), no. 1, 1-12.

[18] M. Naeem, S. Khan, and F. M. Sakar, Faber polynomial coefficients estimates of bi-univalent functions, Inter. J. Maps Math., 3 (2020), no. 1, $57-67$.

[19] N. Magesh and S. Bulut, Chebyshev polynomial coefficient estimates for a class of analytic bi-univalent functions related to pseudo-starlike functions, Afr. Mat., 29 (2018), no. 1-2, 203-209.

[20] N. Magesh and J. Yamini, Fekete-Szegö problem and second Hankel determinant for a class of bi-univalent functions, Tbilisi Math. J., 11 (2018), no. $1,141-157$.

[21] H. Orhan, N. Magesh and C. Abirami, Fekete-Szegö problem for biBazilevič functions related to shell-like curves, AIMS Mathematics, $\mathbf{5}$ (2020), no. 5, 4412-4423. 
[22] S. Porwal and S. Kumar, New subclasses of bi-univalent functions defined by multiplier transformation, Stud. Univ. Babeş-Bolyai Math., 65 (2020), no. $1,47-55$.

[23] F. M. Sakar and S. M. Aydogan, Initial bounds for certain subclasses of generalized Sălăgean type bi-univalent functions associated with the Horadam polynomials, J. Quality. Measur. Anal., 15 (2019), no. 1, 89100.

[24] G. Singh, G. Singh and G. Singh, A subclass of bi-univalent functions defined by generalized Sãlãgean operator related to shell-like curves connected with Fibonacci numbers, Int. J. Math. Math. Sci., 2019, Art. ID $7628083,1-7$.

[25] H. M. Srivastava, Altınkaya and S. Yalçın, Certain subclasses of biunivalent functions associated with the Horadam polynomials, Iran. J. Sci. Technol. Trans. A Sci., 43 (2019), no. 4, 1873-1879.

[26] H. M. Srivastava, F. M. Sakar and H. Özlem Güney, Some general coefficient estimates for a new class of analytic and bi-univalent functions defined by a linear combination, Filomat, 32 (2018), no. 4, 1313-1322.

[27] H. M. Srivastava, S. S. Eker, S. G. Hamidi and J. M. Jahangiri, Faber polynomial coefficient estimates for bi-univalent functions defined by the Tremblay fractional derivative operator, Bull. Iranian Math. Soc., 44 (2018), no. 1, 149-157.

[28] H. M. Srivastava, A. K. Mishra and P. Gochhayat, Certain subclasses of analytic and bi-univalent functions, Appl. Math. Lett., 23 (2010), no. 10, 1188-1192.

[29] H. M. Srivastava, A. Motamednezhad and E. A. Adegani, Faber polynomial coefficient estimates for bi-univalent functions defined by using differential subordination and a certain fractional derivative operator, Mathematics, 8 (2020), 172, 1-12.

[30] A. K. Wanas and A. L. Alina, Applications of Horadam polynomials on Bazilevic bi-univalent function satisfying subordinate conditions, IOP Conf. Series: J. Phy., 1294 (2019), 032003, 1-6. 